\title{
Caractérisation de la race bovine Somba à l'aide de marqueurs moléculaires
}

\author{
K. Moazami-Goudarzi ${ }^{1 *}$ D.M.A. Belemsaga ${ }^{2}$ \\ G. Ceriotti ${ }^{3}$ D. Laloë ${ }^{4}$ F. Fagbohoun ${ }^{5}$ \\ N'T. Kouagou ${ }^{6}$ I. Sidibé ${ }^{2}$ V. Codjia ${ }^{7}$ \\ M.C. Crimella ${ }^{3}$ F. Grosclaude ${ }^{1}$ S.M. Touré ${ }^{2}$
}

\begin{abstract}
Mots-clés
Bovinae - Somba - Zébu - Polymorphisme génétique - Microsatellite Phylogénie - Afrique occidentale.
\end{abstract}

\begin{abstract}
Résumé
Le polymorphisme de quatre catégories de marqueurs du génome -11 systèmes de groupes sanguins, 5 locus des protéines du lait, 2 locus de protéines sanguines et 33 microsatellites, soit au total 51 locus - a été analysé dans quatre populations ou « races » bovines d'Afrique de l'Ouest : les races taurines Somba et Lagunaire, la population de zébus Peuls soudanais et la population Borgou, qui provient du métissage entre taurins et zébus, en vue de caractériser le polymorphisme de la race Somba et d'évaluer sa distance génétique avec les trois autres populations, notamment la race Lagunaire avec laquelle elle présente une forte ressemblance phénotypique. Quelles qu'aient été les catégories de marqueurs ou les méthodes utilisées, les quatre populations se sont séparées nettement les unes des autres. Au niveau des groupes sanguins, les différences les plus nettes ont été observées entre les taurins et les zébus, notamment dans les systèmes $A, B$ et $S$. On a retrouvé par ailleurs chez les zébus la forte fréquence des allèles $\mathrm{Alb}^{\mathrm{S}}$ et $\mathrm{Hb}^{\mathrm{B}}$, ainsi que la prédominance bien connue de I'haplotype $\alpha_{\mathrm{s} 1}-\mathrm{Cn}^{\mathrm{C}}, \beta-\mathrm{Cn}^{\mathrm{A} 2}, \kappa-\mathrm{Cn}^{\mathrm{A}}$ qui contraste avec celle de l'haplotype $\alpha_{\mathrm{s} 1^{-}}$ $\mathrm{Cn}^{\mathrm{B}}, \beta-\mathrm{Cn}^{\mathrm{A} 1}, \kappa-\mathrm{Cn}^{\mathrm{B}}$ chez les taurins africains. Au niveau des microsatellites, I'analyse factorielle des correspondances a souligné le rôle discriminant de I'allèle ETH $225^{139}$, dont la fréquence a été très élevée chez la race Somba, et celui des allèles Hel $13^{182}$ et INRA $037^{114}$ qui ont paru spécifiques respectivement des zébus et de la race Lagunaire. Les fréquences de ces allèles dans la population Borgou ont été sensiblement intermédiaires entre celles des zébus et celles des taurins. Dans le cadre d'une démarche visant à établir dans quelle mesure la connaissance du génotype d'un animal aux 33 marqueurs microsatellites permettait d'identifier sa population d'origine, une proportion de 97 p. 100 d'animaux bien classés a été obtenue, les erreurs de classement s'étant limitées à des zébus incorrectement qualifiés de Borgou et vice versa.
\end{abstract}

\section{INTRODUCTION}

Les premiers bovins domestiques ayant peuplé l'Afrique ont été des animaux sans bosse, introduits dans ce continent par l'Egypte, à partir de populations originaires d'Asie du Sud-Ouest. Ces taurins appartenaient à deux grands groupes : des animaux à cornes

\footnotetext{
1. Laboratoire de Génétique biochimique et de cytogénétique, Inra, 78352 Jouy-enJosas Cedex, France

2. Cirdes, 01 BP 454, Bobo-Dioulasso 01, Burkina Faso

3. Istituto Zootecnica, via G. Celoria 10, 20133 Milano, Italia

4. Station de Génétique quantitative et appliquée, Inra, 78352 Jouy-en-Josas Cedex, France

5. Laboratoire vétérinaire Bohicon, BP 2069, Bénin

6. BP 114, Sokodé / BP 91, Acavet, Niantougou, Togo

7. Direction de l'Elevage, BP 03-2900, Cotonou, Bénin

* Auteur pour la correspondance

Tél : +33 (0)1 34652572 ; fax : +33 (0)1 34652478 ; goudarzi@jouy.inra.fr
}

longues, déjà présents en Egypte 5000 ans avant J.-C., et des animaux à cornes courtes, arrivés environ 2000 ans avant J.-C. La présence de bovins à bosse provenant du Moyen-Orient a été attestée en Egypte dès le Nouvel Empire (environ 1500 ans avant J.-C.), mais l'expansion du zébu en Afrique s'est faite bien plus tard, avec l'invasion arabe (vers l'an 700 de l'ère chrétienne). Mieux adaptés aux conditions arides, les zébus se sont implantés, souvent par métissage, à la place des populations taurines préexistantes. Toutefois, en Afrique de l'Ouest, les taurins ont pu se maintenir dans les zones humides infestées par les glossines grâce à leur trypanotolérance, aptitude dont les zébus sont dépourvus. Néanmoins, un certain degré de métissage avec les zébus est décelable dans bon nombre de populations taurines (19) et les zébus continuent à gagner du terrain sur les taurins compte tenu de l'évolution des écosystèmes.

On retrouve dans l'Afrique de l'Ouest actuelle les deux types taurins mentionnés ci-dessus (figure 1). Le type à cornes longues n'est représenté que par la race N'Dama (le terme de race est utilisé dans 


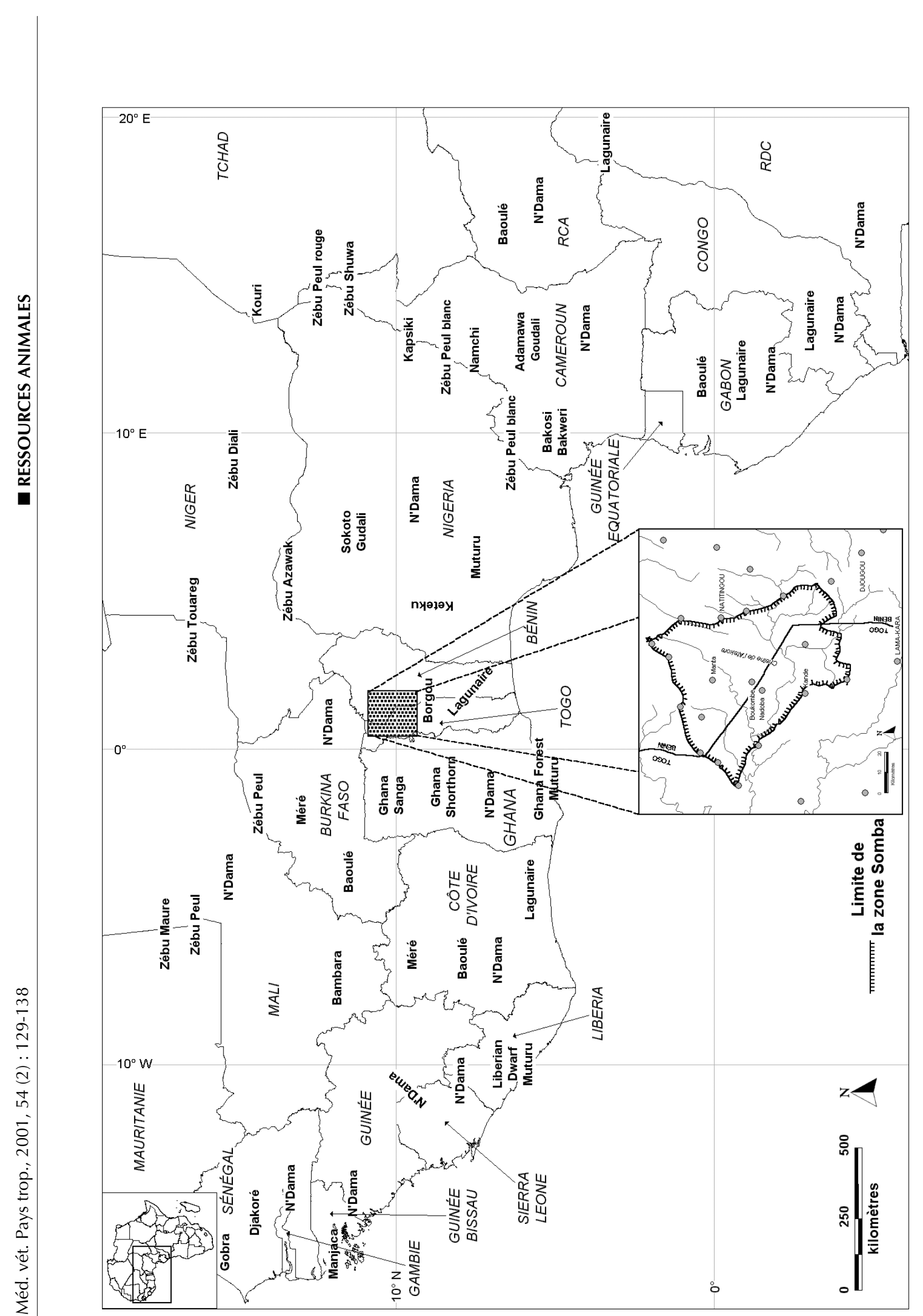


cet article même s'il ne recouvre pas les mêmes réalités qu'en Europe) qui, compte tenu de ses meilleures aptitudes zootechniques, s'est largement répandue au-delà de son berceau, la Guinée, et représente environ la moitié des effectifs taurins de la zone (34). Les taurins à cornes courtes se répartissent en une douzaine de races différentes, classées en deux grands groupes : les races naines (hauteur au garrot : 80 à $90 \mathrm{~cm}$ ) qui se rencontrent surtout dans les milieux humides, les forêts et les zones côtières, et les races de savane (hauteur au garrot : 90 à $110 \mathrm{~cm}$ ). Selon Rege et coll. (34), deux races prédominent largement, avec des effectifs estimés à 600 000-870 000 têtes, la race Baoulé et la race Shorthorn du Ghana, qui appartiennent toutes deux au type de savane. A l'opposé, plusieurs races (Bakweri, Bakosi, Kapsiki, Namchi et Liberian Dwarf Muturu) ne comptent plus qu'un à quelques milliers de têtes.

La race Somba, qui se rattache au type de savane, se rencontre dans un territoire recouvrant le nord-ouest du Bénin (région Tammari) et le nord-est du Togo (région Tamberma) (figure 1). Les éleveurs l'utilisent généralement pour la culture attelée, mais elle a surtout fait l'objet d'une exploitation du type "cueillette », les animaux étant utilisés en paiement de dots ou abattus à l'occasion de cérémonies rituelles animistes. Ses effectifs estimés ont chuté d'environ 75000 en 1977 à 26000 en 1997, par suite d'une mauvaise gestion des troupeaux (abattages des animaux les plus performants) et surtout du fait de croisements de plus en plus fréquents avec les zébus, notamment dans les troupeaux de Peuls, où cohabitent des zébus, des animaux Somba et leurs produits de croisement.

Cette chute d'effectifs, si elle persistait, condamnerait la race à plus ou moins brève échéance. Cette étude s'est inscrite dans le cadre d'un projet européen visant à faire le point sur l'environnement, les aptitudes zootechniques et l'originalité génétique de cette race, afin d'étudier la pertinence d'une politique de préservation. Elle a eu pour objectif la caractérisation de la race Somba et l'analyse de ses relations avec des populations voisines à l'aide de marqueurs moléculaires polymorphes.

\section{MATERIEL ET METHODES}

\section{Echantillonnages}

Les quatre races retenues dans cette étude ont été les races taurines Somba et Lagunaire, la race du zébu Peul soudanais et la race Borgou qui est une race métisse (zébu x Lagunaire ou zébu x Somba). Les prélèvements d'échantillons individuels de lait et de sang ont été effectués de mai 1997 à avril 2000 dans des troupeaux privés. Compte tenu des contraintes du terrain, les deux types de prélèvements ont été souvent obtenus à partir d'animaux différents. La collecte a porté, au total, sur 281 échantillons de lait (93 Somba, 172 Lagunaires, 16 Borgou) et sur 883 prises de sang destinées à l'analyse des groupes sanguins (478 Somba, 109 Lagunaires, 98 Borgou, 198 zébus Peuls soudanais). Par ailleurs, 201 de ces échantillons de sang (50 Somba, 51 Lagunaires, 50 Borgou, 50 zébus Peuls soudanais) ont servi au typage des microsatellites.

\section{Taurins Somba}

Les animaux de cette étude ont été prélevés dans le berceau de la race. Les trois quarts provenaient du Bénin et les autres du Togo. Au Bénin, les animaux ont été prélevés chez une trentaine de propriétaires de la sous-préfecture de Boukoumbé. Au Togo, les animaux ont été prélevés chez une dizaine de propriétaires du canton de Nadoba. Le nombre d'animaux prélevés par propriétaire a varié de 1 à 5 .

\section{Taurins Lagunaires}

Les animaux provenaient de 17 propriétaires de la région de Porto Novo au Bénin. Le nombre d'animaux prélevés par propriétaire a varié de 1 à 5 .

\section{Zébus Peuls soudanais}

Les échantillons de sang provenaient de cinq propriétaires de la région de Malanville au Bénin. Le nombre d'animaux prélevés par propriétaire a varié de 7 à 14 . Les données concernant le polymorphisme des lactoprotéines du zébu Peul soudanais ont été celles de Mahé et coll. (21). Les échantillons de lait provenaient de troupeaux privés situés aux alentours de Bobo-Dioulasso au Burkina Faso.

\section{Borgou}

Les animaux provenaient de cinq propriétaires du département du Borgou au Bénin. Le nombre d'animaux prélevés par propriétaire a varié de 5 à 20.

\section{Systèmes génétiques polymorphes}

Un total de 51 marqueurs génétiques ont été analysés, incluant 5 locus de protéines du lait, 2 locus de protéines du sang, 11 systèmes de groupes sanguins érythrocytaires et 33 marqueurs microsatellites.

\section{Protéines du lait}

Les techniques d'analyses des caséines $\alpha_{\mathrm{s} 1}, \beta$ et $\kappa$, de l' $\alpha$-lactalbumine $(\alpha-\mathrm{La})$ et de la $\beta$-lactoglobuline $(\beta-\mathrm{Lg})$ ont été décrites par Mahé et Grosclaude (20).

\section{Protéines du sang}

Le polymorphisme des protéines sanguines a été analysé par électrophorèse horizontale en gel d'acétate de cellulose [albumine sérique (Alb), hémoglobine $(\mathrm{Hb})]$. Les techniques ont été décrites par Queval et Petit (31) et Queval et Bambara (29).

\section{Groupes sanguins}

Les 11 systèmes de groupes sanguins érythrocytaires connus chez les bovins (A, B, C, F, J, L, M, S, Z, R', T') ont été analysés par isohémolyse (14). Dans les études antérieures, le polymorphisme des deux systèmes de groupes sanguins les plus complexes, les systèmes $B$ et $C$, vraisemblablement codés par plusieurs gènes étroitement liés, avaient été simplifiés et ramenés à ce qui pouvait être assimilé à de véritables séries alléliques $(13,30)$. C'est ainsi que le système $B$ avait été réduit aux six allèles obtenus en regroupant les phénogroupes (haplotypes) comportant respectivement les facteurs antigéniques $G_{1}, I_{1}, K, P$, T et $J$ ", et à un septième allèle regroupant les phénogroupes résiduels. Dans les races étudiées dans le présent travail, l'allélisme de certains de ces facteurs n'est pas toujours vérifié. Toutefois, pour permettre les comparaisons avec les études antérieures, les fréquences retenues ont été celles des facteurs $\mathrm{G}_{1}, \mathrm{I}_{1}, \mathrm{~K}$, $\mathrm{P}, \mathrm{T}$ et J', et celle de l'allèle résiduel négatif. Le système $\mathrm{C}$ a été réduit aux quatre allèles obtenus par regroupement des phénogroupes comportant respectivement les facteurs antigéniques $\mathrm{C}_{1}, \mathrm{C}_{2}$, $\mathrm{C}{ }_{1}$ et $\mathrm{C}{ }_{2}$; un cinquième allèle, négatif pour ces quatre facteurs antigéniques et déjà identifié par Queval et coll. (30), a été observé et retenu chez la race Lagunaire (allèle « négatif »).

Les 65 allèles pris en compte pour les systèmes de groupes sanguins et les protéines du sang et du lait sont présentés dans le tableau I.

\section{Microsatellites}

Dans cette étude 33 marqueurs microsatellites ont été utilisés : INRA 063, INRA 005, ETH 225, IlSTS 005, HEL 5, HEL 1, INRA 035, ETH 152, INRA 023, ETH 10, HEL 9, CSSM 66, INRA 032, ETH 3, BM 2113, BM 1824, HEL 13, INRA 037, BM 1818, ILSTS 006, MM 12, CSRM 60, ETH 185, HAUT 24, HAUT 27, TGLA 227, TGLA 126, TGLA 122, TGLA 53, 


\section{Tableau I}

Allèles détectés pour les systèmes de groupes sanguins, les protéines du sang et les lactoprotéines

\begin{tabular}{|c|c|c|c|c|c|c|c|c|c|c|c|c|c|c|c|c|c|c|c|}
\hline & \multicolumn{11}{|c|}{ Groupes érythrocytaires } & \multicolumn{8}{|c|}{ Protéines } \\
\hline & A & B & C & $\mathbf{F}$ & J & $\mathbf{L}$ & $\mathbf{M}$ & $\mathbf{S}$ & $\mathbf{Z}$ & $\mathbf{R}^{\prime}$ & $\mathbf{T}^{\prime}$ & Al & $\mathrm{Hb}$ & $\alpha_{s 1}-C n$ & $\beta-C n$ & $\kappa-C n$ & $\alpha-$ La & $\beta-\operatorname{Lg}$ & Total \\
\hline \multirow[t]{18}{*}{ Allèles } & $A Z^{\prime}$ & $\left(G_{1}\right)$ & $\left(\mathrm{C}_{1}\right)$ & $\mathrm{F}$ & $J$ & $\mathrm{~L}$ & M1 & $\mathrm{SH}^{\prime}$ & Z & $\mathrm{R}^{\prime}$ & $\mathrm{T}^{\prime}$ & A & A & B & $A^{1}$ & A & A & A & \\
\hline & A & $\left(I_{1}\right)$ & $\left(C_{2}\right)$ & V & - & - & $M^{\prime}$ & $\mathrm{SS}^{\prime \prime} \mathrm{H}^{\prime}$ & - & - & - & B & B & C & $\mathrm{A}^{2}$ & B & B & B & \\
\hline & - & $(\mathrm{K})$ & $\left(C^{\prime \prime}{ }_{1}\right)$ & - & & & - & $\mathrm{SS}^{\prime \prime} \mathrm{U}^{\prime \prime} \mathrm{H}^{\prime}$ & & & & & & $X$ & B & & & & \\
\hline & & $(\mathrm{P})$ & $\left(\mathrm{C}_{2}^{\prime \prime}\right)$ & & & & & $\mathrm{S}^{\prime \prime} \mathrm{UH}^{\prime}$ & & & & & & & & & & & \\
\hline & & $(\mathrm{T})$ & $(-)$ & & & & & $U H^{\prime \prime} \mathrm{H}^{\prime}$ & & & & & & & & & & & \\
\hline & & $\left(J^{\prime \prime}\right)$ & & & & & & $U H^{\prime \prime} S^{\prime \prime} H^{\prime}$ & & & & & & & & & & & \\
\hline & & $\left(\_\right)$ & & & & & & $\mathrm{UH}^{\prime}$ & & & & & & & & & & & \\
\hline & & & & & & & & $\mathrm{U}_{0}^{\prime} \mathrm{U}^{\prime \prime}$ & & & & & & & & & & & \\
\hline & & & & & & & & $\mathrm{H}^{\prime}$ & & & & & & & & & & & \\
\hline & & & & & & & & $U^{\prime}{ }_{1,2}$ & & & & & & & & & & & \\
\hline & & & & & & & & $\mathrm{U}_{1,2}^{\prime} \mathrm{U}^{\prime \prime}$ & & & & & & & & & & & \\
\hline & & & & & & & & $U_{2}^{\prime}$ & & & & & & & & & & & \\
\hline & & & & & & & & $U_{2}^{\prime} U^{\prime \prime}$ & & & & & & & & & & & \\
\hline & & & & & & & & $\mathrm{H}^{\prime \prime} \mathrm{H}^{\prime}$ & & & & & & & & & & & \\
\hline & & & & & & & & $\mathrm{S}^{\prime \prime} \mathrm{H}^{\prime}$ & & & & & & & & & & & \\
\hline & & & & & & & & $U_{0}^{\prime}$ & & & & & & & & & & & \\
\hline & & & & & & & & $\mathrm{U}^{\prime \prime} \mathrm{H}^{\prime}$ & & & & & & & & & & & \\
\hline & & & & & & & & $H^{\prime \prime} U^{\prime \prime} H^{\prime}$ & & & & & & & & & & & \\
\hline $\begin{array}{l}\text { Nb. } \\
\text { d'allèles }\end{array}$ & 3 & 7 & 5 & 3 & 2 & 2 & 3 & 18 & 2 & 2 & 2 & 2 & 2 & 3 & 3 & 2 & 2 & 2 & 65 \\
\hline
\end{tabular}

SPS 115, INRA $\kappa$, INRA 016, INRA 072. Les 30 premiers marqueurs sont ceux de la liste de référence internationale. Les trois derniers ont été choisis pour relier les quatre populations de l'étude aux études précédentes de Moazami-Goudarzi et coll. (24) et de Souvenir Zafindrajaona et coll. (39). Le choix de ces marqueurs a été effectué en fonction, d'une part, de leurs caractéristiques techniques (bonne aptitude à l'amplification et interprétation aisée des typages) et, d'autre part, de leurs caractéristiques génétiques (nombre d'allèles, localisation et répartition dans le génome). L'analyse regroupant les races françaises et africaines a été effectuée sur un total de neuf microsatellites (INRA 063, INRA 005, ETH 225, INRA 035, ETH 152, INRA 032, INRA $\kappa$, INRA 016, INRA 072). L'extraction d'Adn génomique a été effectuée selon le protocole de Jeanpierre (15). Les séquences des amorces des microsatellites et les modalités de détermination des génotypes par électrophorèse en gel de polyacrylamide-urée après Pcr en présence d'amorces fluorescentes sont décrites dans la base de données intitulée Cattle Diversity Database (http://www.ri.bbsrc.ac.uk/cdiv) et dans Moazami-Goudarzi et coll. (24).

\section{Traitement des données}

\section{Calcul des fréquences alléliques}

Pour les systèmes génétiques à allèles codominants, les fréquences alléliques ont été établies par comptage direct. Pour les locus bialléliques comportant un allèle négatif, les fréquences alléliques ont été estimées par la méthode de la racine carrée et, pour les systèmes complexes, par la méthode d'itération de Ceppellini et coll. (5).

\section{Test du rapport de vraisemblance}

L'hypothèse d'égalité de fréquences alléliques par race et par marqueur a été testée par le test du rapport de vraisemblance avec la procédure Freq du progiciel SAS (36).

\section{Taux d'hétérozygotie}

Le taux d'hétérozygotie théorique non biaisé $\mathrm{H}$ a été calculé d'après Nei (26) à l'aide du programme Genepop (version 3.1) (33).

\section{Equilibre de Hardy-Weinberg}

La conformité de la structure des populations à l'équilibre de Hardy-Weinberg a été vérifiée par un test exact à l'aide du programme Genepop (version 3.1) (33).

\section{F-statistique}

La structuration des populations a été analysée par le calcul des trois coefficients $\mathrm{F}_{\mathrm{IS}}, \mathrm{F}_{\mathrm{ST}}$ et $\mathrm{F}_{\mathrm{IT}}$ (41). Ces différents coefficients sont utilisés pour désigner la variabilité génétique aux niveaux des individus (I), des sous-populations (S) et de la population totale (T). Le programme Genepop (version 3.1) (33) a été utilisé pour effectuer ces calculs.

\section{Analyse factorielle des correspondances}

L'analyse factorielle des correspondances (Afc) $(16,17)$ est un procédé d'analyse multidimensionnelle analogue à l'analyse des composantes principales, mais adaptée à l'étude des liaisons entre deux ou plusieurs variables qualitatives. La méthode présentée ici est une analyse sur tableaux juxtaposés de contingence (18). 
L'analyse des correspondances s'effectue sur un tableau particulier, formé de tableaux de contingence accolés. Les lignes du tableau correspondent aux races et les colonnes aux allèles. Cette analyse est équivalente à une analyse des correspondances multiples « race systèmes alléliques » où les différents systèmes génétiques sont indépendants (17). Le principal intérêt de la méthode est de représenter les races par un système de points situés dans un espace euclidien, ce qui permet la visualisation des regroupements des races. De plus, elle autorise une représentation simultanée des races et des allèles. Ces points peuvent être projetés sur les différents axes et plans factoriels, en bénéficiant de tous les outils d'aide à l'interprétation (pourcentage d'inertie, contributions à la construction de l'axe, qualité de la projection sur les axes, etc.) qu'offre ce type d'analyse.

Cette méthode peut également s'utiliser dans un contexte de discrimination ou d'affectation : la race est au milieu du nuage de points représentants les individus. Dans ce contexte, cette méthode est appelée « analyse discriminante barycentrique » (17). Ces analyses ont été réalisées avec le progiciel SAS (procédure Corresp). Théoriquement, quel que soit le contexte d'utilisation (typologie des races, discrimination ou affectation des animaux), la méthode conduit aux mêmes résultats. Néanmoins, la gestion des données manquantes est différente selon le contexte et les animaux qui ne sont pas typés pour l'ensemble des marqueurs ne peuvent être inclus dans l'analyse discriminante barycentrique, alors qu'ils le sont dans le premier type d'analyse.

\section{Calcul des distances génétiques}

Les deux distances génétiques, dont l'emploi est préconisé par Takezaki et Nei (40), ont été utilisées pour quantifier la dissemblance globale entre les races : la distance de Cavalli-Sforza et Edwards (4), ou $\mathrm{D}_{\mathrm{C}}$, et la distance standard de Nei (25), ou $\mathrm{D}_{\mathrm{S}}$. Les calculs ont été effectués à l'aide du logiciel Phylip (12).

\section{Construction des phénogrammes}

La représentation arborée de neighbor-joining (35) et l'algorithme dit « du lien moyen » (Upgma) (38) ont été utilisés pour construire les phénogrammes à partir de la matrice des distances. La robustesse des arbres a été analysée par la méthode du bootstrap $(7,11)$. Après 500 ré-échantillonnages des marqueurs, un arbre de consensus majoritaire permettant de visualiser les concordances a été construit (23). Les calculs ont été effectués à l'aide du logiciel Phylip (12).

\section{Affectation}

Pour déterminer la race des individus à partir de leur génotype à un ensemble de locus, deux méthodes de discrimination, ou d'affectation, ont été appliquées. Ces deux méthodes sont fondées sur le maximum de vraisemblance.

La méthode des fréquences (27) calcule en fonction du génotype de chaque individu sa probabilité d'appartenance aux différentes races et l'affecte à la race pour laquelle cette probabilité est maximale. Les locus sont supposés être indépendants et les populations être à l'équilibre de Hardy-Weinberg.

La deuxième méthode (32) utilise une approche bayésienne. Les vraisemblances d'appartenance des individus aux populations sont calculées en utilisant une distribution a priori uniforme des fréquences alléliques.

Ces deux méthodes ont été appliquées avec la procédure du leave one out. Cette procédure ne tient pas compte de l'animal à affecter pour calculer les paramètres des populations.

Le jeu de données a porté sur 201 animaux (50 Somba, 51 Lagunaires, 50 Borgou, 50 zébus Peuls Soudanais) dont le génotype aux 33 microsatellites était connu. Les calculs ont été effectués à l'aide du programme Geneclass (6).

\section{RESULTATS ET DISCUSSION}

Le test du rapport de vraisemblance a conclu à l'inégalité des fréquences alléliques entre races, quel qu'ait été le marqueur analysé, à un niveau de significativité inférieur à 1 p. 100. Les races étudiées étaient donc statistiquement différentes.

En ce qui concernait les groupes sanguins, les principales différences entre taurins et zébus ont été observées au niveau des systèmes $\mathrm{A}, \mathrm{B}$ et $\mathrm{S}$. Dans le système $\mathrm{A}$, a été retrouvée chez les taurins la très forte prédominance, déjà signalée par Braend (3), de l'allèle A dont la fréquence a varié de 0,92 à 1 selon les échantillons considérés, alors que ce système a été franchement polymorphe chez les zébus (fréquence de l'allèle AZ' de l'ordre de 0,1 à 0,15 et fréquence de l'allèle " négatif », ou " a », de l'ordre de 0,30 à 0,50 ). On peut considérer la fréquence très élevée de l'allèle A chez les taurins comme une attestation du prolongement en Afrique du gradient de fréquence observé par Grosclaude et coll. (13) chez les races ouest-européennes, la fréquence de cet allèle augmentant du nord vers le sud. Dans le système B, la somme des fréquences des allèles comportant les antigènes de référence $\left(G_{1}\right.$, $\mathrm{I}_{1}, \mathrm{~K}, \mathrm{P}_{2}, \mathrm{~T}$ ', J') a été nettement plus élevée chez les zébus $(0,5$ à $0,7)$ que chez les taurins, où elle n'a atteint que 0,2 dans la population Somba et seulement 0,01 (valeur exceptionnellement basse) dans la population Lagunaire. Dans le système $S$, enfin, la fréquence de l'allèle U' a été nettement plus élevée chez les taurins $(0,36$ à 0,57$)$ que chez les zébus (environ 0,10$)$, cet écart ayant été notamment compensé par une fréquence plus élevée de l'allèle H' chez les zébus. En ce qui concernait les protéines du sang, a été retrouvée chez les zébus la forte fréquence des allèles $\mathrm{Alb}^{\mathrm{S}}$ et $\mathrm{Hb}^{\mathrm{B}}$ déjà mise en évidence par la synthèse de Baker et Manwell (2) et le travail de Queval et coll. (30). Enfin, les observations faites sur le polymorphisme des caséines ont concordé avec celle de Mahé et coll. (21), l'haplotype $\alpha_{\mathrm{s} 1}-\mathrm{Cn}^{\mathrm{B}}, \beta-\mathrm{Cn}^{\mathrm{A} 1}, \kappa-\mathrm{Cn}^{\mathrm{B}}$ prédominant nettement chez les taurins africains (tableau II), par opposition à l'haplotype $\alpha_{\mathrm{s} 1}-\mathrm{Cn}^{\mathrm{C}}, \beta-\mathrm{Cn}^{\mathrm{A} 2}, \kappa-\mathrm{Cn}^{\mathrm{A}}$ majoritaire chez les zébus (21). Ces résultats confirment une fois de plus que les gènes des caséines font partie des marqueurs permettant une bonne discrimination entre Bos taurus et Bos indicus, même si les différences de fréquences entre les deux sous-espèces ne sont pas aussi accusées que pour d'autres marqueurs (figure 2).

Tous les microsatellites ont été polymorphes chez les quatre races (tableau III). Le nombre total d'allèles par race a été compris entre 156 pour la race Lagunaire et 280 pour la race Borgou. Il a atteint 337 pour l'ensemble des races. L'examen des fréquences alléliques a révélé des profils de répartition très différents suivant les microsatellites et les races. Pour certains marqueurs, des allèles ont été très fréquents chez une race et pratiquement absents chez les autres. Soixante-six allèles n'ont été détectés que dans une seule des quatre races étudiées, mais la fréquence de ces allèles «privés » a toujours été inférieure à 5 p. 100. Sur les 337 allèles détectés, 144 (43 p. 100) ont eu une fréquence inférieure à 5 p. 100 et 39 ont eu des fréquences très différentes, d'une part, entre les taurins et, d'autre part, entre les zébus et les Borgou. C'est le cas de l'allèle 107 du microsatellite Hel 1 qui a atteint une fréquence de 56 p. 100 chez le zébu Peul soudanais, de 24 p. 100 chez le Borgou et qui n'a pas été détecté chez les taurins Somba et Lagunaire. Les fréquences alléliques du Borgou se sont généralement situées entre celles du zébu et des taurins (par exemple, l'allèle 185 du microsatellite CSSM 66 a eu une fréquence de 70 p. 100 chez les Lagunaires, de 49 p. 100 chez les Somba, de 35 p. 100 chez les Borgou et de 25 p. 100 chez les zébus).

Le taux d'hétérozygotie par race a varié entre 0,54 pour la race Lagunaire et 0,73 pour la race Borgou (tableau III). Ces résultats ont concordé avec ceux de MacHugh et coll. (19) qui, en se 


\section{Tableau II}

Fréquences alléliques aux locus des cinq lactoprotéines et haplotypiques aux locus $\alpha_{\mathrm{s} 1^{-}}, \beta-$, $\kappa$-Casein

\begin{tabular}{|c|c|c|c|c|}
\hline $\begin{array}{l}\text { Fréquences } \\
\text { alléliques }\end{array}$ & & $\begin{array}{c}\text { Somba } \\
(93)\end{array}$ & $\begin{array}{l}\text { Lagunaire } \\
\text { (172) }\end{array}$ & $\begin{array}{c}\text { Borgou } \\
\text { (16) }\end{array}$ \\
\hline \multirow{3}{*}{$\alpha_{s 1}-C n$} & B & 0,87 & 0,92 & 0,53 \\
\hline & C & 0,13 & 0,08 & 0,44 \\
\hline & $x$ & 0 & 0 & 0,03 \\
\hline \multirow[t]{4}{*}{$\beta-C n$} & $A^{1}$ & 0,64 & 0,57 & 0,25 \\
\hline & $A^{2}$ & 0,36 & 0,43 & 0,75 \\
\hline & B & 0 & 0 & 0 \\
\hline & D & 0 & 0 & 0 \\
\hline \multirow[t]{3}{*}{$\kappa-C n$} & A & 0,25 & 0,42 & 0,59 \\
\hline & B & 0,75 & 0,58 & 0,41 \\
\hline & $\mathrm{J}$ & 0 & 0 & 0 \\
\hline \multirow{2}{*}{$\alpha$-La } & A & 0,01 & 0,07 & 0,19 \\
\hline & B & 0,99 & 0,93 & 0,81 \\
\hline \multirow[t]{2}{*}{$\beta-\operatorname{Lg}$} & A & 0,23 & 0,42 & 0,13 \\
\hline & B & 0,77 & 0,58 & 0,87 \\
\hline
\end{tabular}

\begin{tabular}{lll} 
Haplotypes & Somba & Lagunaire \\
\hline $\mathrm{BA}^{1} \mathrm{~A}$ & 1 & 0,04 \\
$\mathrm{BA}^{1} \mathrm{~B}$ & 0,54 & 0,52 \\
$\mathrm{BA}^{2} \mathrm{~A}$ & 0,08 & 0,3 \\
$\mathrm{BA}^{2} \mathrm{~B}$ & 0,15 & 0,05 \\
$\mathrm{BA}^{2} \mathrm{~J}$ & 0 & 0 \\
$\mathrm{BBA}$ & 0 & 0 \\
$\mathrm{BBB}$ & 0 & 0 \\
$\mathrm{BDB}$ & 0 & 0 \\
$\mathrm{CA}{ }^{1} \mathrm{~A}$ & 0 & 0,01 \\
$\mathrm{CA}^{1} \mathrm{~B}$ & 0 & 0 \\
$\mathrm{CA}^{2} \mathrm{~A}$ & 0,08 & 0,07 \\
$\mathrm{CA}^{2} \mathrm{~B}$ & 0,05 & 0,01 \\
& & \\
& &
\end{tabular}

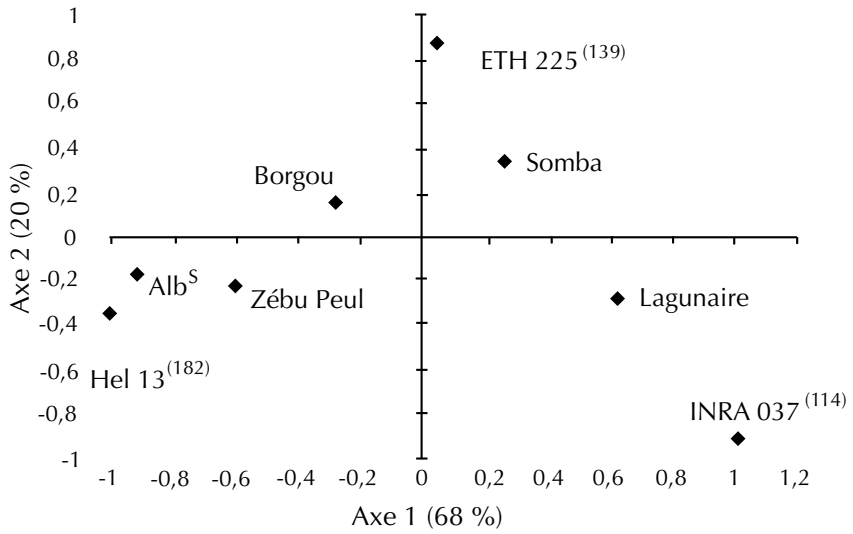

Figure 2 : analyse factorielle des correspondances (groupes sanguins, protéines du sang et du lait et 33 microsatellites).

fondant sur l'analyse du polymorphisme de 20 microsatellites, ont obtenu des taux d'hétérozygotie de 0,63 pour les zébus d'Afrique de l'Ouest et de 0,54 pour le N'Dama.

Le taux d'hétérozygotie par microsatellite a varié de 0,37 (SPS 115, INRA $\kappa$ à 0,87 (BM2113), avec une valeur moyenne de 0,71 . Ces résultats sont du même ordre de grandeur que ceux de Schmid et coll. (37) qui, sur la base des 30 microsatellites de la liste internationale, obtiennent pour cinq races bovines suisses un taux d'hétérozygotie de 0,70 .

D'après les résultats des statistiques de F, 11,4 p. 100 de la variation génétique totale $\left(\mathrm{F}_{\mathrm{ST}}\right)$ s'expliquaient par des différences entre races et 88,6 p. 100 par des différences au sein d'une même race. Chez les races européennes, ces différences entre races sont généralement plus faibles puisque le degré de différenciation génétique des sous-populations $\left(\mathrm{F}_{\mathrm{ST}}\right)$ varie de 7 à 10 p. $100(19,24,36)$.

Sur 132 tests, 16 écarts significatifs $(\mathrm{P}<0,01)$ à l'équilibre de Hardy-Weinberg ont été détectés. Ils concernaient les quatre races analysées et 11 microsatellites (tableau III). Ces déséquilibres pou- vaient s'expliquer par l'existence vraisemblable d'un certain degré de consanguinité dans ces populations à faible effectif. D'autres hypothèses peuvent être avancées, sans pouvoir être vérifiées, comme la proximité de microsatellites avec des gènes sélectionnés ou encore la présence de microsatellites dans des régions en déséquilibre de liaison dans le génome bovin (9).

L'analyse factorielle des correspondances effectuée sur l'ensemble des 51 marqueurs sépare les quatre races (figure 2). Le premier axe, qui rend compte de 68 p. 100 de l'inertie, oppose taurins et zébus. Au vu de la position du Borgou, entre le zébu et le Somba et plus éloignée du Lagunaire, on peut supposer que cette population est issue de croisements entre le zébu et le Somba. Les allèles contribuant le plus à la construction de cet axe sont l'allèle $S$ de l'albumine et l'allèle 182 du microsatellite HEL 13. La fréquence de ces allèles chez le Borgou se situe entre celle des zébus et celle des taurins. Leur contribution est dix fois plus élevée que la contribution moyenne des allèles.

Le deuxième axe, qui rend compte de 20 p. 100 de l'inertie, distingue le Lagunaire du Somba. Les allèles contribuant le plus à la construction de cet axe sont l'allèle 139 du microsatellite ETH 225 et l'allèle 114 du microsatellite INRA 037 qui est présent quasi exclusivement chez la race Lagunaire. Leur contribution est également dix fois plus élevée que la contribution moyenne des allèles.

Pour vérifier si chacun des 51 marqueurs structure ces quatre populations de façon identique, des Afc ont été effectuées en les considérant individuellement. Mis à part les trois systèmes T', ETH 3 et SPS 115, la même typologie, caractérisée par une position intermédiaire du Borgou entre le zébu et les taurins, a été observée. Ces résultats ont montré une bonne robustesse de la représentation moyenne.

Des analyses supplémentaires ont été réalisées en considérant deux sous-ensembles de marqueurs, les 33 microsatellites d'une part, les protéines et les groupes sanguins d'autre part. La même typologie a été retrouvée dans les deux cas.

L'Afc effectuée en considérant chaque animal comme une entité distincte montre une claire différenciation des animaux selon leur 


\section{Tableau III}

Nombre d'allèles $(N)$, taux d'hétérozygotie théorique non biaisé $(H)$, écart aux proportions de Hardy-Weinberg (HW) et F-statistique par race et par microsatellite

\begin{tabular}{|c|c|c|c|c|c|c|c|c|c|c|c|c|c|c|}
\hline \multirow[t]{2}{*}{ Locus } & \multicolumn{3}{|c|}{ Somba } & \multicolumn{3}{|c|}{ Lagunaire } & \multicolumn{3}{|c|}{ Zébu } & \multicolumn{3}{|c|}{ Borgou } & \multicolumn{2}{|c|}{ Total } \\
\hline & $\mathbf{N}$ & $\mathbf{H}$ & HW * & $\mathbf{N}$ & $\mathbf{H}$ & HW* $^{*}$ & $\mathbf{N}$ & $\mathbf{H}$ & $\mathrm{HW}^{*}$ & $\mathbf{N}$ & $\mathbf{H}$ & HW* $^{*}$ & $\mathbf{N}$ & $\mathbf{H}$ \\
\hline BM 1818 & 6 & 0,82 & & 6 & 0,68 & 0,006 & 9 & 0,84 & & 9 & 0,81 & & 9 & 0,83 \\
\hline BM 1824 & 5 & 0,54 & & 3 & 0,30 & & 5 & 0,77 & & 6 & 0,73 & & 8 & 0,64 \\
\hline BM 2113 & 7 & 0,75 & & 7 & 0,73 & & 9 & 0,83 & & 9 & 0,86 & & 12 & 0,87 \\
\hline TGLA 53 & 10 & 0,79 & & 9 & 0,74 & & 13 & 0,84 & & 15 & 0,89 & & 18 & 0,86 \\
\hline TGLA 122 & 8 & 0,54 & & 5 & 0,62 & & 8 & 0,65 & & 12 & 0,67 & & 14 & 0,72 \\
\hline TGLA 126 & 7 & 0,76 & & 5 & 0,49 & & 8 & 0,74 & & 7 & 0,78 & & 8 & 0,75 \\
\hline TGLA 227 & 10 & 0,83 & & 6 & 0,66 & & 8 & 0,62 & & 10 & 0,83 & & 12 & 0,83 \\
\hline HEL 1 & 5 & 0,43 & & 5 & 0,48 & & 6 & 0,64 & & 6 & 0,70 & & 7 & 0,67 \\
\hline HEL 5 & 6 & 0,72 & & 5 & 0,73 & & 7 & 0,75 & 0,000 & 8 & 0,80 & 0,004 & 8 & 0,79 \\
\hline HEL 9 & 9 & 0,73 & & 4 & 0,66 & & 10 & 0,82 & & 11 & 0,88 & & 11 & 0,84 \\
\hline HEL 13 & 4 & 0,44 & & 3 & 0,45 & & 5 & 0,59 & & 6 & 0,67 & & 6 & 0,65 \\
\hline ETH 3 & 10 & 0,79 & 0,001 & 5 & 0,75 & & 9 & 0,79 & 0,009 & 9 & 0,78 & 0,001 & 14 & 0,80 \\
\hline ETH10 & 7 & 0,52 & 0,001 & 3 & 0,46 & & 6 & 0,77 & & 8 & 0,80 & & 8 & 0,71 \\
\hline ETH 152 & 4 & 0,48 & & 2 & 0,42 & & 5 & 0,60 & & 5 & 0,68 & & 6 & 0,67 \\
\hline ETH 185 & 6 & 0,50 & 0,000 & 6 & 0,38 & & 10 & 0,81 & 0,008 & 10 & 0,57 & & 13 & 0,61 \\
\hline ETH 225 & 7 & 0,77 & & 5 & 0,62 & & 8 & 0,74 & & 10 & 0,81 & & 12 & 0,82 \\
\hline HAUT 24 & 7 & 0,81 & & 8 & 0,75 & & 8 & 0,81 & & 8 & 0,81 & & 11 & 0,83 \\
\hline HAUT 27 & 6 & 0,60 & & 3 & 0,54 & & 6 & 0,73 & & 8 & 0,79 & & 8 & 0,70 \\
\hline MM 12 & 5 & 0,47 & & 6 & 0,33 & & 11 & 0,83 & & 13 & 0,82 & & 13 & 0,71 \\
\hline ILSTS 005 & 4 & 0,54 & & 3 & 0,56 & & 5 & 0,73 & & 5 & 0,74 & & 5 & 0,69 \\
\hline ILSTS 006 & 6 & 0,59 & 0,007 & 4 & 0,65 & & 9 & 0,64 & & 8 & 0,70 & & 11 & 0,68 \\
\hline INRA 005 & 5 & 0,56 & & 5 & 0,46 & & 6 & 0,61 & & 4 & 0,62 & & 7 & 0,58 \\
\hline INRA 016 & 11 & 0,80 & & 4 & 0,61 & & 11 & 0,78 & & 10 & 0,76 & & 14 & 0,80 \\
\hline INRA 023 & 8 & 0,58 & & 4 & 0,47 & & 9 & 0,67 & & 9 & 0,77 & 0,004 & 12 & 0,67 \\
\hline INRA 032 & 6 & 0,54 & & 2 & 0,02 & & 10 & 0,76 & & 10 & 0,76 & & 12 & 0,64 \\
\hline INRA 035 & 2 & 0,31 & & 3 & 0,52 & & 5 & 0,59 & & 5 & 0,42 & & 6 & 0,49 \\
\hline INRA 037 & 7 & 0,67 & & 7 & 0,73 & & 10 & 0,78 & & 7 & 0,82 & & 11 & 0,82 \\
\hline INRA 063 & 6 & 0,69 & & 6 & 0,72 & & 7 & 0,71 & 0,000 & 6 & 0,57 & & 8 & 0,72 \\
\hline INRA 072 & 8 & 0,79 & & 4 & 0,68 & & 9 & 0,78 & 0,000 & 13 & 0,87 & 0,000 & 14 & 0,83 \\
\hline INRA K & 3 & 0,07 & & 3 & 0,09 & & 6 & 0,62 & & 6 & 0,54 & & 10 & 0,37 \\
\hline SPS 115 & 5 & 0,33 & & 3 & 0,28 & & 6 & 0,44 & 0,002 & 7 & 0,40 & & 7 & 0,37 \\
\hline CSSM 66 & 8 & 0,68 & & 4 & 0,45 & 0,007 & 9 & 0,83 & & 11 & 0,81 & & 12 & 0,75 \\
\hline CSSM 60 & 8 & 0,76 & & 8 & 0,67 & & 8 & 0,42 & & 9 & 0,59 & & 10 & 0,73 \\
\hline Total & 216 & & & 156 & & & 261 & & & 280 & & & 337 & \\
\hline Moyenne & 6,5 & 0,61 & & 4,7 & 0,54 & & 7,9 & 0,71 & & 4,5 & 0,73 & & 10 & 0,71 \\
\hline
\end{tabular}

F-Statistique $-\mathrm{F}_{\mathrm{ST}}: 0,114 ; \mathrm{F}_{\mathrm{IS}}: 0,076 ; \mathrm{F}_{\mathrm{IT}}: 0,174$

$* \mathrm{P}<0,01$

race (figure 3). Seul un zébu se situe dans le nuage correspondant aux Borgou. Il s'agit du seul zébu possédant l'allèle 117 du microsatellite TGLA 126. Or, cet allèle a une fréquence de 12 p. 100 chez les Borgou et est pratiquement inexistant chez les autres races. Les méthodes d'affectation de Paetkau et coll. (27) et de Rannala et Mountain (32) ont été appliquées à l'ensemble des 201 animaux caractérisés sur les 33 microsatellites. Le pourcentage d'animaux bien classés a été de 98 p. 100 pour la première méthode et de 97 p. 100 pour la seconde. Les erreurs de classement ont concerné des animaux zébus classés comme Borgou et inversement. Les animaux Somba ont tous été correctement affectés. A l'inverse, aucun animal non Somba n'a été affecté chez cette race. Ces résultats affirment très nettement l'identité génétique de la race Somba et contredisent ainsi les travaux d'Aboagye et coll. (1). Ces auteurs se fondant sur des ressemblances morphologiques et des homologies entre fréquences alléliques de l'hémoglobine et

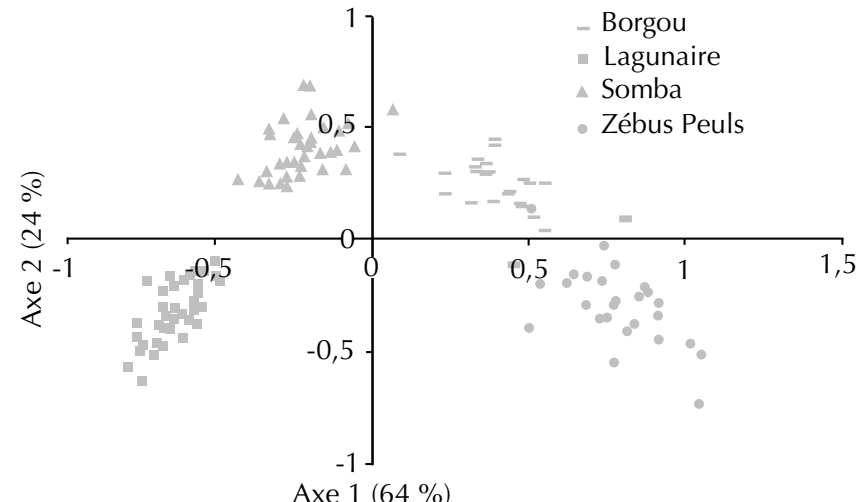

Figure 3 : analyse factorielle des correspondances à partir de 33 microsatellites. Projection des individus sur le premier plan factoriel. 
de certains facteurs érythrocytaires avaient conclu à l'identité des populations Somba et Lagunaire, qui n'étaient donc censées différer que par leur localisation géographique.

Neuf microsatellites étant communs à cette étude et aux travaux antérieurs de Souvenir et coll. (39) et de Moazami-Goudarzi et coll. (24), les données des trois sources ont été regroupées afin d'étendre l'analyse à d'autres populations locales d'Afrique de l'Ouest (Kouri, N'Dama, Baoulé, zébu Malgache, zébu Choa, zébu M'Bororo) et d'Europe (Charolaise, Limousine, Vosgienne, Montbéliarde, Parthenaise, Maine-Anjou, Holstein, Normande, Bretonne Pie noire, Jersey). La même typologie a été obtenue quelle qu'ait été la distance génétique $(4,25)$ ou la méthode d'agrégation (neighbor-joining, Upgma) utilisées.

La figure 4 représente, à titre d'exemple, le phénogramme obtenu à partir du polymorphisme des neuf microsatellites en utilisant la distance de Cavalli-Sforza et Edwards (4) et la méthode d'agrégation du neighbor-joining (35). Même si le nombre de marqueurs microsatellites utilisés a été relativement faible, les résultats obtenus ont été très semblables aux données historiques et aux résultats de la littérature $(8,10,22,28,30)$. On constate en particulier une séparation très marquée entre populations africaines et européennes (valeur de bootstrap : 95 p. 100), ainsi qu'une forte opposition entre taurins et zébus. Parmi les taurins africains, la population N'Dama, rattachée au type à cornes longues, se retrouve isolée des populations à cornes courtes qui, elles, sont regroupées. Le sous-ensemble des populations de zébus est plus structuré, et ceci sur des bases visiblement géographiques. Il se scinde en effet en populations du Burkina Faso et du Bénin d'une part (zébus Peuls et métis Borgou), et en populations du Tchad d'autre part

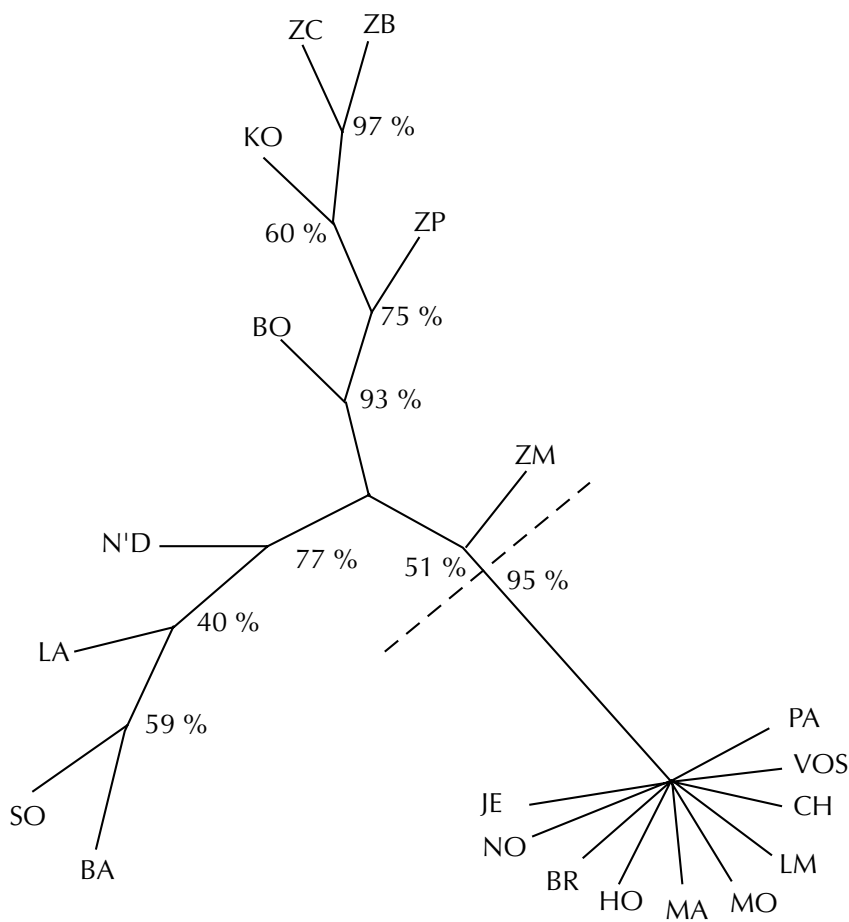

Figure 4 : arbre de consensus obtenu après 500 rééchantillonnages des données $(9$ microsatellites, distance de Cavalli-Sforza et Edwards, méthode de neighbor-joining).

BA : Baoulé ; BO : Borgou ; KO : Kouri ; LA : Lagunaire ; $N^{\prime} D$ : N'Dama ; $S O$ : Somba ; ZB : zébu M'Bororo; ZC : zébu Choa ; ZM : zébu Malgache; ZP : zébu Peul; $B R$ : Bretonne Pie noire; $\mathrm{CH}$ : Charolaise ; $\mathrm{HO}$ : Holstein ; JE : Jersey ; LM : Limousine ; MA : Maine-Anjou ; MO : Montbéliarde; NO : Normande ; PA : Parthenaise ; VOS : Vosgienne. (zébu Choa, zébu M'Bororo et « taurins » Kouri). Rappelons que selon les résultats de Souvenir et coll. (39), la race Kouri, classée habituellement parmi les taurins, montre des traces de métissage très significatives avec le zébu, conformément avec ce que l'on sait de l'histoire de cette population originale. Enfin, le zébu Malgache est isolé des autres zébus africains et forme à lui seul une branche à part. Ce résultat est cohérent avec le statut insulaire de cette population qui pourrait par ailleurs provenir d'animaux amenés directement d'Inde par l'océan Indien, alors que, sur le continent africain, les zébus ont été introduits par l'Egypte.

\section{CONCLUSION}

Les résultats de la présente étude sont tout à fait cohérents avec ceux des travaux antérieurs de Moazami-Goudarzi et coll. (24), Queval et coll. (30) et Souvenir et coll. (39). Les populations de zébus et les populations taurines se séparent nettement et, parmi ces dernières, le groupe des populations à cornes courtes (Baoulé, Lagunaire, Somba) se distingue de la population N'Dama, seule population à cornes longues de cette région à être peu métissée.

L'élément nouveau qui paraît le plus intéressant dans cette étude réside dans le fait que l'utilisation d'un ensemble de 33 marqueurs microsatellites, soit guère plus d'un marqueur par chromosome en moyenne, a permis de distinguer, sans aucune erreur, les animaux Somba des animaux Lagunaires, alors que les deux populations passaient pour être génétiquement très proches, voire identiques. Ce résultat montre que, même si les populations bovines d'Afrique de l'Ouest n'ont pas été délimitées et gérées comme les races européennes et passent pour s'être largement métissées entre elles, de nettes différences génétiques peuvent être mises en évidence sans difficulté entre certaines d'entre elles. Cette conclusion semble justifier la volonté actuelle de préserver les ressources génétiques bovines de l'Afrique de l'Ouest et notamment la race Somba.

\section{Remerciements}

Cette étude a été réalisée dans le cadre du projet « La race bovine Somba : caractérisation et recherche en vue de son amélioration et de sa conservation », financé par l'Union européenne, Commission des communautés européennes, Direction générale du développement (Inco-Dc contrat $n^{\circ}$ Erbic18Ct960031). Ce travail a pu être réalisé grâce à la participation et à la collaboration de nombreuses personnes. Nous tenons ainsi à remercier les éleveurs, les responsables des services de l'Elevage du Bénin et du Togo, les autorités administratives des préfectures de Boukoumbé et Nadoba qui, par leur accueil, ont beaucoup facilité notre tâche sur le terrain. Nous remercions pour leurs collaborations techniques S. Sylla, R. Sangaré, F. Damah-Soundjia (groupes sanguins, variants électrophorétiques) et V. Michel (carte géographique) du Cirdes (Centre international de recherche développement sur l'élevage en zone subhumide, Burkina Faso), M.-F. Mahé (lactoprotéines) du laboratoire de Génétique biochimique et de cytogénétique de l'Inra (Institut national de la recherche agronomique, France), G. Noé (groupes sanguins) du Gie Labogena (Laboratoire d'analyses génétiques pour les espèces animales, France), A. Caroli (élaboration des données) et G. Strillacci (extraction d'Adn, genotypage) de l'Umil.Iz (Universita degli Studi Milano, Istituto di Zootecnica, Italie), P. Agbadjè, B. N'Dah Ntcha, S. Adam Samari (échantillonnage des animaux) du Ppea (Projet promotion de l'élevage dans l'Atacora, Bénin). Nous sommes particulièrement reconnaissants à J.-P. Sero Sandjougouma du Ppea, K. Djabakou, K. Adomefa Dsra-Inzv (Direction scientifique de la recherche agronomique, Institut national zootechnique et vétérinaire, Togo) pour l'intérêt et la contribution qu'ils ont portés à cette étude. 


\section{BIBLIOGRAPHIE}

1. ABOAGYE G.S., TAWAH C.L., REGE J.E.O., 1994. Shorthorn cattle of West and Central Africa. III. Physical, adaptative and special genetic characteristics. Revue mond. Zootech., 78: 22-32.

2. BAKER C.M.A., MANWELL C., 1980. Chemical classification of cattle. 1. Breed groups. Anim. Blood Groups Biochem. Genet., 11: 127-150.

3. BRAEND M., 1979. Blood groups of Nigerian cattle. Comparative aspects. Anim. Blood Groups Biochem. Genet., 10: 49-56.

4. CAVALLI-SFORZA L., EDWARDS W., 1967. Phylogenetic analysis: models and estimation procedures. Evolution, 21: 550-570.

5. CEPPELLINI R., SINISCALCO M., SMITH C.A.B., 1956. The estimation of gene frequencies in a random mating population. Ann. Hum. Genet., 20: $97-115$.

6. CORNUET J.M., PIRY S., LUIKART G., ESTOUP A., SOLIGNAC M., 1999. New methods employing multilocus genotypes to select or exclude populations as origins of individuals. Genetics, 153: 1989-2000.

7. EFRON B., 1979. Bootstrap methods: another look at the jacknife. Ann. Stat., 7: 1-26.

8. EPSTEIN H., 1971. The origin of domestic animals of Africa, Vol. 1. New York, NY, USA, Africana Publishing, p. 208-212.

9. FARNIR F., COPPIETERS W., ARANZ J.J., BERZI P., CAMBISANO N., GRISART B., KARIM L., MARCQ F., MOREAU L., MNI M., NEZER C., SIMON P., VANMANSHOVEN P., WAGENAAR D., GEORGES M., 2000. Extensive genome-wide linkage disequilibrium in cattle. Genome Res., 10: 220-227.

10. FELIUS M., 1995. Cattle breeds: an encyclopedia. Doetinchem, The Netherlands, Misset, p. 498-548.

11. FELSENSTEIN J., 1985. Confidence limits on phylogenies: an approach using the bootstrap. Evolution, 39: 783-791.

12. FELSENSTEIN J., 1993. PHYLIP - Phylogeny inference package, Version 3.5. Seattle, WA, USA, Washington University, Dpt of Genetics.

13. GROSCLAUDE F., AUPETIT R., LEFEBVRE J., MERIAUX J.C., 1990. Essai d'analyse des relations génétiques entre les races bovines françaises à l'aide du polymorphisme biochimique. Genet. Sel. Evol., 22 : 317-338.

14. GROSCLAUDE F., GUERIN G., HOULIER G., 1979. The genetic map of the B system of cattle blood groups as observed in French breeds. Anim. Blood Groups Biochem. Genet., 10: 199-218.

15. JEANPIERRE M., 1987. A rapid method for purification of DNA from blood. Nucl. Acid Res., 15: 9611.

16. LEBART L., MORINEAU A., TABART N., 1977. Techniques de la description statistique. Paris, France, Dunod, $351 \mathrm{p}$.

17. LEBART L., MORINEAU A., PIRON M., SHRIVER M.D., LOFTUS R.T., 1995. Statistique exploratoire multidimensionnelle. Paris, France, Dunod, $439 \mathrm{p}$

18. LECLERC A., 1975. L'analyse des correspondances sur juxtaposition de tableaux de contingence. Revue Stat. appl., 23 : 5-16.

19. MACHUGH D.E., SHRIVER M.D., LOFTUS R.T., CUNNINGHAM P., BRADLEY D.G., 1997. Microsatellite DNA variation and the evolution, domestication and phylogeography of taurine zebu cattle (Bos taurus and Bos indicus). Genetics, 146: 1071-1086.

20. MAHE M.F., GROSCLAUDE F., 1993. Polymorphism of $\beta-C n$ in the creole goat of Guadeloupe: evidence for a null allele. Genet. Sel. Evol., 25: $403-408$

21. MAHE M.F., MIRANDA G., QUEVAL R., BADO A., SOUVENIR ZAFINDRAJAONA P., GROSCLAUDE F., 1999. Genetic polymorphism of milk proteins in African Bos taurus and Bos indicus populations. Characterization of variants $\alpha_{\mathrm{s} 1}-\mathrm{Cn} \mathrm{H}$ and $\kappa-\mathrm{Cn}$ J. Genet. Sel. Evol., 31: 239-253.
22. MANWELL C., BAKER C. M.A., 1980. Chemical classification of cattle. 2. Phylogenic tree and specific status of the zebu. Anim. Blood Groups Biochem. Genet., 11: 151-162.

23. MARGUSH T., MCMORRIS F.R., 1981. Consensus N-trees. Bull. Math. Biol., 43: 239-244.

24. MOAZAMI-GOUDARZI K., LALOE D., FURET J.P., GROSCLAUDE F., 1997. Analysis of genetic relationships between 10 cattle breeds with 17 microsatellites. Anim. Genet., 28: 338-345.

25. NEI M., 1972. Genetic distance between populations. Am. Nat., 106 283-292.

26. NEI M., 1978. Estimation of average heterozygosity and genetic distance from a small number of individuals. Genetics, 89: 583-590.

27. PAETKAU D., CALVERT W., STIRLING I., STROBECK C., 1995. Microsatellite analysis of population structure in Canadian polar bears. Mol. Ecol., 4: 347-354.

28. PAYNE W.J.A., HODGES H., 1997. Tropical cattle origins, breeds and breeding policies. Oxford, UK, Blackwell Science, p. 47-70, 135-156.

29. QUEVAL R., BAMBARA L., 1984. Le polymorphisme de l'albumine dans la race Baoulé et une population de zébus de type soudien. Revue Elev. Méd. vét. Pays trop., 37 ( $\mathrm{n}^{\circ}$ spécial) : 288-296.

30. QUEVAL R., MOAZAMI-GOUDARZI K., LALOE D., MERIAUX J.C., GROSCLAUDE F., 1998. Relations génétiques entre populations de taurins ou zébus d'Afrique de l'Ouest et taurins européens. Genet. Sel. Evol., 30 : 367-383.

31. QUEVAL R., PETIT J.P., 1982. Polymorphisme biochimique de I'hémoglobine de populations bovines trypanosensibles, trypanotolérantes et leur croisement dans l'Ouest africain. Revue Elev. Méd. vét. Pays trop., 35 : 137-146.

32. RANNALA B., MOUNTAIN J.L., 1997. Detecting immigration by using multilocus genotypes. Proc. natl Acad. Sci., 94: 9197-9201.

33. RAYMOND M., ROUSSET F., 1995. An exact test for population differentiation. Evolution, 49: 1280-1283.

34. REGE J.E.O., ABOAGYE G.S., TAWAH C.L., 1994. Shorthorn cattle of West and Central Africa. I. Origin, distribution, classification and population statistics. Revue mond. Zootech., 78: 2-13.

35. SAITOU N., NEI M., 1987. The neighbour-joining method: a new method for reconstructing phylogenetic trees. Mol. Biol. Evol., 4: 406-425.

36. SAS, 1989. SAS user's guide: statistics, Version 6, P-846. Cary, NC, USA, SAS Institute.

37. SCHMID M., SAITBEKOVA N., GAILLARD C., DOLF G., 1999 Genetic diversity in Swiss cattle breeds. J. Anim. Breed. Genet., 116: 1-8.

38. SNEATH P.H.A., SOKAL R.R., 1973. Numerical taxonomy. San Francisco, CA, USA, WH Freeman.

39. SOUVENIR ZAFINDRAJAONA P., ZEUH V., MOAZAMIGOUDARZI K., LALOË D., BOURZAT D., IDRISS A., GROSCLAUDE F., 1999. Etude du statut phylogénétique du bovin Kouri du lac Tchad à I'aide de marqueurs moléculaires. Revue Elev. Méd. vét. Pays trop., 52 : 155-162.

40. TAKEZAKI N., NEI M., 1996. Genetic distances and reconstruction of phylogenetic trees from microsatellite DNA. Genetics, 144: 389-399.

41. WEIR B.S., COCKERHAM C.C., 1984. Estimating F-statistics for the analysis of population structure. Evolution, 38: 1358-1370.

Reçu le 20.07.2001, accepté le 20.12.2001 


\section{Summary}

Moazami-Goudarzi K., Belemsaga D.M.A., Ceriotti G., Laloë D., Fagbohoun F., Kouagou N'T., Sidibé I., Codjia V., Crimella M.C., Grosclaude F., Touré S.M. Characterization of Somba Cattle Breed Using Molecular Markers

The polymorphism of four categories of genome markers11 blood group systems, 5 lactoprotein loci, 2 blood protein loci and 33 microsatellites, i.e. 51 loci in total-was analyzed in four cattle populations or "breeds" from West Africa: the Somba and Lagoon taurine breeds, the Sudanese zebu Peul population and the Borgu population, crossbred between taurine and zebu. The aim of the study was to characterize the polymorphism of the Somba breed and to evaluate its genetic distance with the other three populations, especially the Lagoon breed, with which it shares a high phenotypical resemblance. Whatever the category of markers or the method used, the four populations were clearly separated. Based on the blood group systems, the most marked differences were observed between taurine and zebu breeds especially for the A, B and S systems. The typical high allele frequencies of $\mathrm{Alb}^{\mathrm{S}}$ and $\mathrm{Hb}^{\mathrm{B}}$ were observed in zebus, as well as the well-known predominance of the $\alpha_{s 1}-C^{C}, \beta-C n^{A 2}, \kappa-$ $\mathrm{Cn}^{\mathrm{A}}$ haplotype, in contrast with the $\alpha_{\mathrm{s} 1}-\mathrm{C} n^{\mathrm{B}}, \beta-\mathrm{Cn}^{\mathrm{A} 1}, \kappa-\mathrm{Cn}^{\mathrm{B}}$ haplotype of African taurines. Based on microsatellites, reciprocal averaging highlighted the discriminating effect of ETH $225^{139}$ allele, whose frequency was very high in the Somba breed, and Hel $13^{182}$ and INRA 037114, which were apparently specific to zebu and Lagoon breeds, respectively. These allele frequencies in the Borgu population were roughly intermediate between those in zebus and taurine breeds. The study also tried to determine whether it was possible to identify the population of origin based on the knowledge of the 33-microsatellite genotype of an animal. Results showed that $97 \%$ of the animals were correctly classified; errors concerned zebus incorrectly classified as Borgus and vice versa.

Key words: Bovinae - Somba - Zebu cattle - Genetic polymorphism - Microsatellite - Phylogeny - West Africa.

\section{Resumen}

Moazami-Goudarzi K., Belemsaga D.M.A., Ceriotti G., Laloë D., Fagbohoun F., Kouagou N'T., Sidibé I., Codjia V., Crimella M.C., Grosclaude F., Touré S.M. Caracterización de la raza bovina Somba, mediante marcadores moleculares

Se analizó el polimorfismo de cuatro categorías de marcadores del genoma-11 sistemas de grupos sanguíneos, 5 locus de proteínas de la leche, 2 locus de proteínas sanguíneas y 33 microsatélites, sea un total de 51 locus-en cuatro poblaciones o « razas » bovinas de Africa del Oeste : las razas taurinas Somba y Lagunar, la población de cebúes Peuls sudaneses y la población Borgu, proveniente de un cruce de taurinos y cebúes, esto con el fin de caracterizar el polimorfismo de la raza Somba y de evaluar su distancia genética con respecto a las otras tres poblaciones, principalmente la raza Lagunar, con la cual presenta un fuerte parecido fenotípico. Con cualesquiera que hayan sido las categorías de marcadores o los métodos utilizados, las cuatro poblaciones se distanciaron claramente las unas de las otras. Al nivel de grupos sanguíneos, las diferencias más marcadas fueron observadas entre los taurinos y los cebúes, sobre todo en los sistemas A, B y S. Se encontró además, en los cebúes, una fuerte frecuencia de alelos $\mathrm{Alb}^{\mathrm{S}}$ y $\mathrm{Hb}^{\mathrm{B}}$, así como la predominancia bien conocida del haplotipo $\alpha_{s 1}-C n^{C}, \beta-C n^{A 2}, \kappa-C n^{A}$ que contrasta con la del haplotipo $\alpha_{s 1}-\mathrm{Cn}^{\mathrm{B}}, \beta-\mathrm{Cn}^{\mathrm{A} 1}, \kappa-\mathrm{Cn}^{\mathrm{B}}$ en los taurinos africanos. Al nivel de los microsatélites, el análisis factorial de correspondencias mostró el papel discriminante del alelo ETH $225^{139}$, cuya frecuencia fue muy elevada en la raza Somba y el de los alelos Hel $13^{182}$ e INRA $037^{114}$ que parecieron específicos para los cebúes y la raza lagunar, respectivamente. Las frecuencias de estos alelos en la población Borgu fueron significativamente intermediarias entre las de los cebúes y las de los taurinos. En el marco de un proceso cuyo objeto fue el de establecer en que medida el conocimiento del genotipo de un animal con 33 marcadores microsatélites permite la identificación de su población de origen, se obtuvo una proporción de $97 \%$ de animales bien clasificados, los errores de clasificación se limitaron a cebúes incorrectamente calificados de Borgu y vice versa.

Palabras clave: Bovinae - Somba - Cébu - Polimorfismo genético - Microsatelite - Filogenía - Africa occidental. 